Article

\title{
Evaluation of a Balloon-Type Vaginal Endoscope Based on Three-Dimensional Printing Technology for Self-Assessment of Pelvic Organ Prolapse
}

\author{
Myoungjae Jun ${ }^{1}\left(\mathbb{D}\right.$, Hieyong Jeong $\left.{ }^{2, *,+} \mathbb{(}\right)$, Masayuki Endo ${ }^{3, *} \mathbb{C}$, Michiko Kodama ${ }^{4}(\mathbb{C}$ \\ and Yuko Ohno ${ }^{3}$ iD \\ 1 Department of Robotics \& Design for Innovative Healthcare, Graduate School of Medicine, \\ Osaka University, 1-7 Yamadaoka, Suita, Osaka 565-0871, Japan; 21cnehemiah@gmail.com \\ 2 School of Electronic \& Computer Engineering, Chonnam National University, 77 Yongbongro, Bukgu, \\ Gwangju 61186, Gwangju, Korea \\ 3 Division of Health Sciences, Graduate School of Medicine, Osaka University, 1-7 Yamadaoka, Suita, \\ Osaka 565-0871, Japan; ohno@sahs.med.osaka-u.ac.jp \\ 4 Department of Obstetrics and Gynecology, Graduate School of Medicine, Osaka University, 1-7 Yamadaoka, \\ Suita, Osaka 565-0871, Japan; michikokodama51@gmail.com \\ * $\quad$ Correspondence: h.jeong@jnu.ac.kr (H.J.); endo@gyne.med.osaka-u.ac.jp (M.E.); Tel.: +82-62-530-3427 (H.J.); \\ +81-6-6879-2533 (M.E.) \\ † Current address: Room 710 in 6-EB, 77 Yongbongro, Bukgu, Gwangju, Gwangju 61186, Korea.
}

Received: 25 June 2020; Accepted: 17 July 2020; Published: 24 July 2020

Featured Application: This work is helpful for ostergard's urogynecology and pelvic floor dysfunction.

\begin{abstract}
Pelvic organ prolapse (POP) can occur if the support tissues or the pelvic floor muscles are weakened and damaged. There is increased probability for POP occurrence after childbirth, menopause, or in overweight women. Because the natural history and progression of POP is still unknown, the approaches used to prevent it have not been clear. POP is an uncomfortable condition that affects one every three women. However, most people feel uncomfortable to discuss it. Herein, we conducted a feasibility evaluation study for self-assessment approaches with a vaginal endoscope based on three-dimensional (3D) printing. The proposed endoscope has two parts: (a) rubber material used to cover it for its intended insertion, to avoid direct contact with the walls of the vagina, and (b) two types of sensors at the tip for measurements. The condition inside the vagina was observed with a camera and depth sensors based on the regulation of the amount of air. Arbitrary temporary prolapses from the testbed's generator enabled us to perceive the location of the problem and symptoms that were regarded as the early stage. As discussed, the low-cost design of the 3D-printed-based vaginal endoscope provides a self-check capability and allows continuous observations that help prevent POP.
\end{abstract}

Keywords: balloon-type vaginal endoscope; three-dimensional printing technology; pelvic organ prolapse

\section{Introduction}

Pelvic organs include the bladder, uterus, and rectum. These organs are held in place by tissues called fascia and ligaments. These tissues help join our pelvic organs to the bony side walls and hold them in a stable manner inside the pelvis. Stability is also provided inferiorly by the pelvic floor muscles. If the fascia and ligaments are torn or stretched for any reason, and if our pelvic floor muscles 
are weakened, our pelvic organs may not be held in their proper location, and they may bulge into the vagina. Thus, pelvic organ prolapses (POPs) can be defined as downward displacements of pelvic organs that result in herniations into or through the vagina or anal canal [1]. Because the prevalence of POP increases with age, the changing demographics of the world's population will result in an increased number of affected women. Based on projections from the United States Census Bureau, the number of American women with ages of 65 or higher will double in the next 25 years to more than 40 million women by 2030 [2]. Based on estimates, the demand for healthcare services related to pelvic floor disorders will increase [3]. The lifetime risk that a woman in the United States will undergo surgery for prolapse or urinary incontinence is $11 \%$, with almost one third of the surgeries represented by repeated procedures. Although the overall rate of prolapse surgery has decreased, this represents a substantial drop in the rate of surgery for women with ages less than 50 years old and a moderate increase for women aged 50 years and higher. The direct cost of prolapse surgery is higher than $\$ 1$ billion per year [4].

The pelvic organs are held inside the pelvis by muscular, healthy fascia. They are held up by the pelvic floor muscles that work like a firm muscle sling. If the support tissues that keep the bladder, uterus, and bowel in place inside the pelvis, or if the pelvic floor muscles are weakened and damaged, prolapse may occur. Childbirth is the main critical cause of prolapse $[5,6]$. On the way down to the vagina, the baby can stretch and tear the support tissues and the pelvic floor muscles. The more vaginal births women have, the more likely it is for them to experience a prolapse. Other things that press down on the pelvic organs and the pelvic floor muscles that can lead to prolapse are persistent coughing, such as a smoker's cough or asthma, heavy lifting, constipation, and straining induced in the effort to empty the bowel [7-10].

There are a few signs that are indicative of a prolapse [11-13]. These signs depend on the type of prolapse and on how much the pelvic organ support has diminished [14-16]. When the prolapse is in inferior anatomical positions, most women can notice something different, such as a heavy feeling or dragging in the vagina, the sensation that a tissue/organ or a lump is moving toward the vagina, or the sensation of a lump bulging out of their vagina. When patients see or feel lumps in the shower, or when they have a bath, or if sexual problems associated with pain or diminished sensation have arisen, it is likely that the bladder may not be empty, the urine stream may be weak, and urinary tract infections may reappear. It is also likely that it may be hard for the patients to empty their bowels. These signs can be worse at the end of the day, but symptoms may subside after lying down. If the prolapse bulges right outside the body, soreness and bleeding may occur as the prolapse rubs on the underwear.

The essential physical examination checks whether the bladder or rectum is shifted when the stomach is strengthened while lying down. The same examination must be performed while standing. Although many systems have been described for staging prolapse, the standard is the system approved by the International Continence Society known as the Pelvic Organ Prolapse Quantification (POP-Q) system [17]. This system measures nine locations in the vagina and vulva relative to the hymen. These nine locations are useful for assigning a stage of prolapse at its most unique site. This method promises to quantitatively evaluate the protrusions of the anterior and posterior walls based on the hymen. The state of prolapse is classified in 5 stages to determine the appropriate treatment [17-19]. Accordingly, the conventional examination is dependent on human observation of medical doctors without any point-of-care device in healthcare.

Most women think that POP is too shameful to describe symptoms to medical doctors. Because avulsion (levator ani muscle) is diagnosed by palpation in conjunction with prolapse quantification, most women are reluctant to be treated actively even if they suffer from POP [20]. Although it is difficult to evaluate the state of prolapse with exact statistical data because most women who suffer from it do not receive active physical examinations, medical doctors estimate that 1 out of 3 women have these symptoms, and 1 out of 9 women need surgical management. Surgery does repair the torn or stretched fascia and ligaments. The surgical procedure may involve going through 
the vagina or the tummy [21]. Sometimes, a surgical mesh is placed in the anterior or posterior vaginal walls as an alternative tissue to damaged fascia and ligaments in cases at which the latter are weak or torn [22]. During the healing process, the connective tissues that are formed around the mesh provide strong support. The major problem of surgical management is that women should prevent the prolapse from recurring [23]. The other problem is that surgical limitations include neurological damage even if operational goals are completed [24].

Meanwhile, if the symptoms are observed in mild cases, nonsurgical management can decrease the frequency and severity of the symptoms, delay or avoid surgery, and potentially prevent worsening the effects owing to the prolapse [1]. Nonsurgical management of POP includes adjunct therapy to address concomitant symptoms, pelvic floor muscle training, and pessaries. Prolapse can often be treated without surgery, primarily in mild cases. The simple approach may involve (a) pelvic floor muscle training [25] ([26] planned the training to match the patient's individual needs in conjunction with the advice of a pelvic floor physiotherapist or continence nurse advisor), (b) understanding the etiology of the prolapse and implementation of the required lifestyle changes, (c) maintenance of functional bowel and bladder habits to avoid straining and the use of a pessary [27-30] (a silicone device that fits in the vagina) carefully measured and placed into the vagina to provide inner support for the pelvic organs, to avoid surgery. As we surveyed, there is no proper healthcare device yet for preventing the prolapse from getting worse.

Thus, if there is a method to self-assess symptoms without the need to undergo a physical examination by a medical doctor, this will alleviate patient discomfort and allow the monitoring of symptoms from an early stage to enforce an active POP nonsurgical management approach [1,25-29]. The early finding enables most women to select and insert the proper pessary with the exact position and orientation and then evaluate the effects of nonsurgical management. Therefore, this study performed a feasibility assessment of the self-evaluation of the symptoms through a vaginal endoscope designed based on 3D printing technology. The features for the proposed endoscope comprise two parts: rubber material required to cover and insert the endoscope into the vagina and two types of sensors at its tip for measurements. Experimental results proved the feasibility of the proposed device based on the generation of arbitrary POPs at the testbed.

\section{Materials and Methods}

Figure 1 summarizes the requirements needed to satisfy the safe insertion of the vaginal endoscope and a description of how to inflate the rubber material, as the concept design. The part on the left represents the parts that are inserted in the vagina, and the part on the right shows the balloon-type vaginal endoscope. When women feel some symptoms, some prolapses can be found at early stages. This means that there is an abnormality of muscle fiber tissue in that area. If the location and condition of the symptoms are detected at the early stage, it is possible to cope with POP nonsurgical management, such as pelvic floor muscle exercises, pessary insertion, etc. At that time, the device can be used by the patient to observe how symptoms should meet the requirements of (a) safe insertion and (b) exact recognition of the location and condition.

\subsection{Insertion of Endoscope}

After all the inserted mechanical parts are covered with the rubber material, such as a medical condom (shown as the yellow-colored part in Figure 1 (middle)), the rubber can be inflated by the air supplied from the device. The red button of the device controls the amount of air to regulate the air pressure that is related to the impedance of the material. The pressured air can be delivered through the device, in which the red dotted arrow indicates the path of the air, as shown in Figure 1 (right side). The material is fixed to the moving part of the endoscope and moves back and forth along the $z$-axis direction based on a manual operation. 


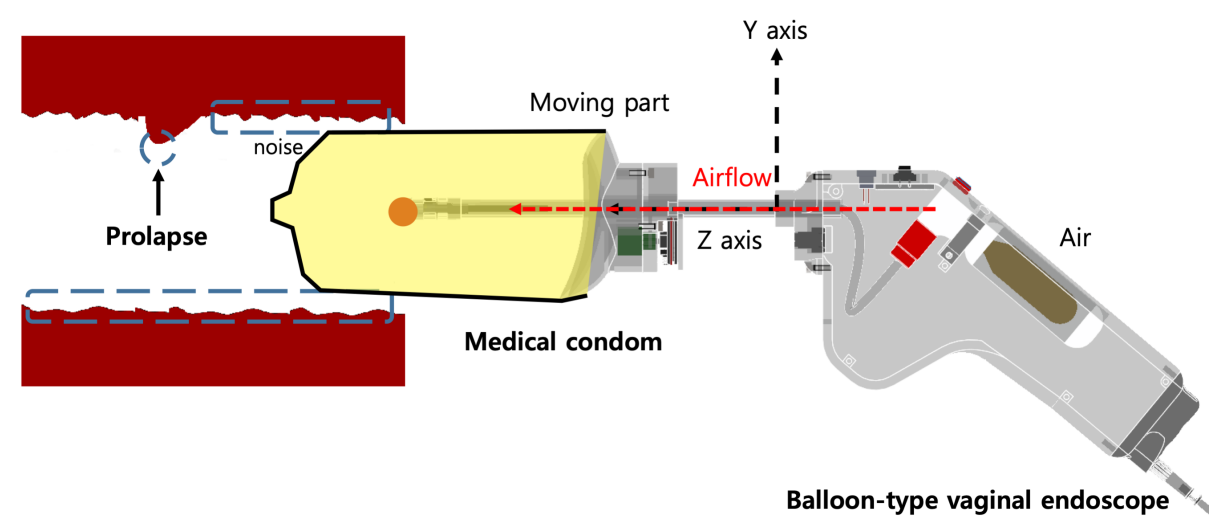

Figure 1. Schematic of concept design in consideration of the requirements for safe insertion in the vaginal endoscope and a depiction of the inflated medical condom to cover the endoscope before insertion.

Because the rubber material protects direct contact between the device and the vaginal wall, it is possible to make sure that there are no significant side effects associated with the use of the condom. Additionally, the advantages of condoms are their low prices, availability without prescription, and little need for planning. Thus, the inflated cover through the rubber material enables the rigid tip of the endoscope to satisfy the safe insertion requirement.

\subsection{Recognition of Location And Symptoms}

\subsubsection{Location of Symptom}

Figure 2 shows the requirements needed to recognize the exact location and condition through the vaginal endoscope, and a description of the experimental system to measure the position, orientation, and condition of symptoms. The directional position along the $z$-axis is computed as follows:

$$
z^{\prime}=d+C_{1}
$$

where the unit of measurement for $z^{\prime}$ is $\mathrm{mm}, C_{1}$ is the constant that is decided by the relation between the offset value for the installation and the tip of the endoscope, and $d$ indicates the measured value acquired by the measuring sensor unit. A distance measuring sensor unit (ST-23G, KOOENSHI Corporation, Republic of Korea) is used, and this sensor is composed of an integrated combination of a position-sensitive detector (PSD, GP2Y0D21YK0F, SHARP Corporation, Japan) that is the infrared-emitting diode (IRED) with signal processing circuit. The allowable range along the $z$-axis direction is $0-140 \mathrm{~mm}$, and the resolution of this sensor unit is $0.5 \mathrm{~mm}$. This sensor unit is installed in the primary gun-type part.

The directional positions along the $\mathrm{x}$ - and $\mathrm{y}$-axes are computed as follows,

$$
\left[\begin{array}{l}
x^{\prime} \\
y^{\prime}
\end{array}\right]=\left[\begin{array}{cc}
\cos (\theta) & -\sin (\theta) \\
\sin (\theta) & \cos (\theta)
\end{array}\right]\left[\begin{array}{c}
0 \\
-C_{2}
\end{array}\right],
$$

where the unit of measurement for $\left[x^{\prime}, y^{\prime}\right]^{T}$ is ${ }^{\circ}, C_{2}$ represents the constant when this is decided at the initial setting, and $\theta$ represents the value measured by the angle sensor. Here, ${ }^{T}$ means the matrix transpose. The used sensor is a hall-angle sensor (P3015-V1-CW360), which can measure the full range of $360^{\circ}$ with a resolution of $0.5^{\circ}$. The miniature noncontact angle sensor P3015 works based on the principle of electromagnetic induction, and the angle is converted to an electrical signal. When the rotation angle of the object is passed to the axis of rotation sensors, the P3015 angle sensor outputs an electrical signal that is proportional to the rotational angle. This green-colored sensor unit is installed in the moving part, as shown in Figure 2. Thus, the calculated values of $\left(x^{\prime}, y^{\prime}, z^{\prime}\right)$ indicate the recognition of the exact location of the symptom that denotes the position and orientation. 


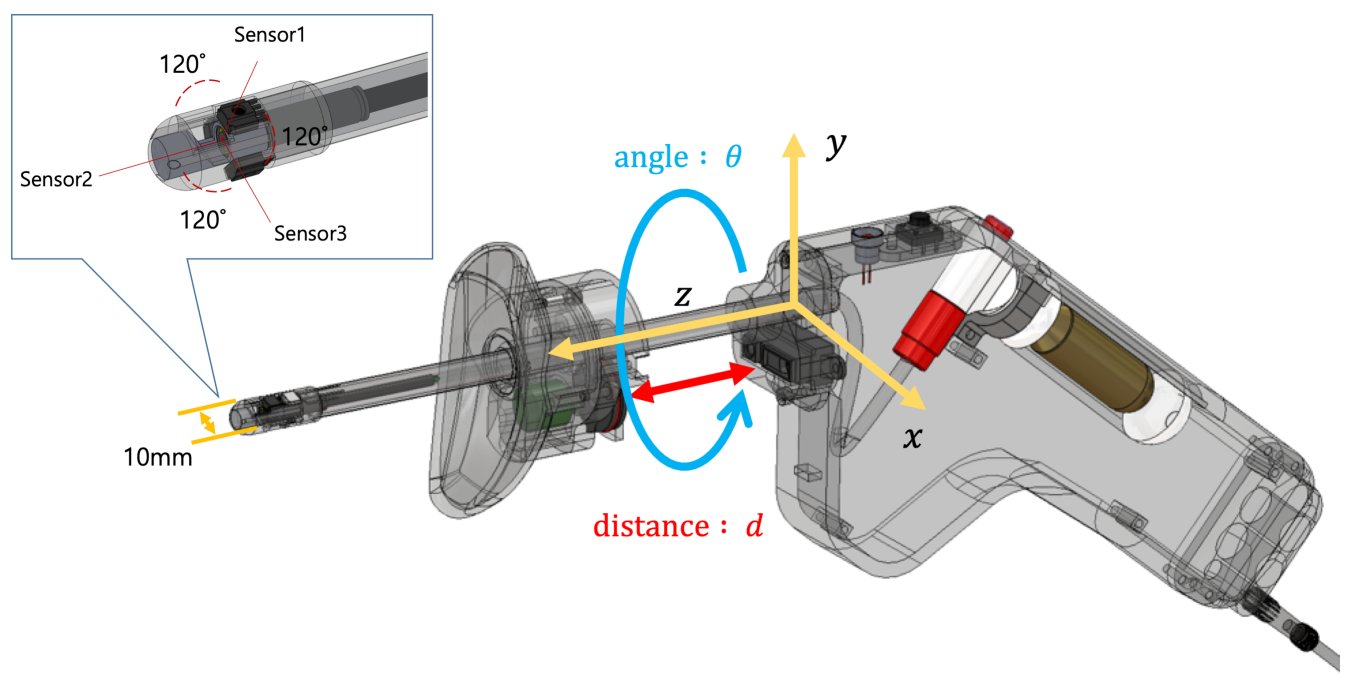

Figure 2. Consideration of requirements to recognize the exact location and condition through the vaginal endoscope and description of the experimental system to measure the position, orientation, and the symptom.

\subsubsection{Conditions of Symptoms}

The conditions of the symptoms can be observed by one camera and three depth sensors, as shown in Figure 2 (left side), which are fixed at the tip of the endoscope. The camera is useful for the understanding of the status of the entire inner parts of the vagina, and three depth sensors help digitize the prolapse with the measured data and the measurement of the size of the POP. The inner diameter of the camera is $3.9 \mathrm{~mm}$, and the product diameter is $4.3 \mathrm{~mm}$. The highest image quality of a $4.3 \mathrm{~mm}$ camera is achieved with HD 720p, with a resolution of $640 \times 480$. The waterproof camera level is IP67. This indicates that the camera is a washable camera and can be washed with water before and after use. The sensor (IS471F) is used to measure the prolapse condition within the range of $0 \sim 20 \mathrm{~mm}$ and with a resolution of $1.0 \mathrm{~mm}$. Although the number of sensors enables us to measure the degree of prolapse without any additional motion, such as rotation, the space is too small to install more than three depth sensors. Three sensors are installed every $120^{\circ}$ as shown in Figure 2.

\section{Design of Experimental System}

\subsection{System Architecture Outside the Endoscope}

Figure 3 shows the system architecture outside the balloon-type vaginal endoscope. This system can be divided into two parts connected with a 6-pin-type connector, namely, in a control box and an endoscope. The control box plays a significant role in the operation of the endoscope. It comprises one power supply for two types of voltages (direct current (DC) $12 \mathrm{~V}$ and DC $5 \mathrm{~V}$ ), an embedded computer system for processing data acquired by the camera and sensors, and two monitors for the display of data from the camera and sensors.

Three types of measured sensing data (depth, distance of $d$, angle of $\theta$ ) are entered through the analog-to-digital (ADC) port and then transformed by the 10-bit ADC converter to a dimensionless value in the range of $0 \sim 1024$. Transformed data are transferred to the single-board computer (Raspberry pi) through serial communication so that the user can confirm the measured data through the graphical user interface (GUI) program. The Raspberry pi sends the measured data to the monitor through the HDMI port, and the camera of the endoscope sends image data with a resolution of 1,080 to the USB C port. 


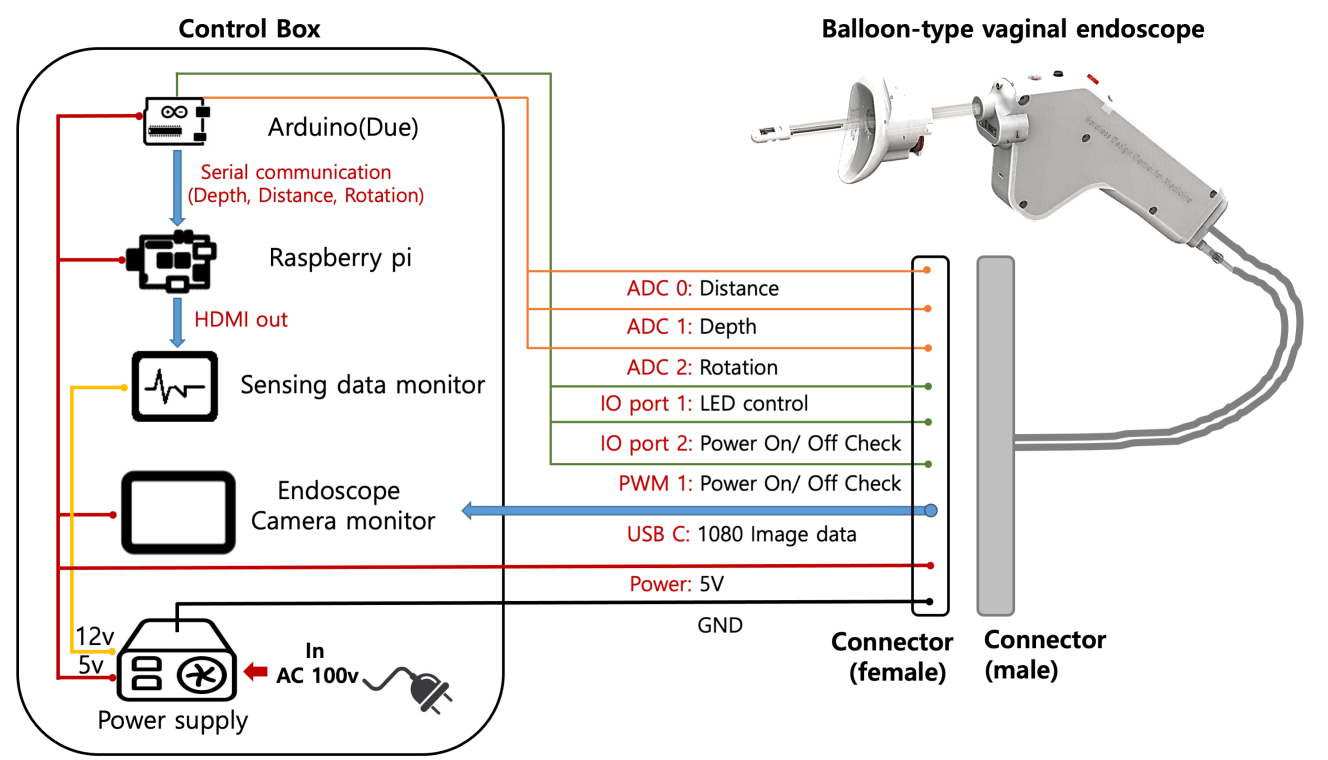

Figure 3. System architecture of the outer parts of the balloon-type vaginal endoscope.

\subsection{Electrical Schematic of the Inner Parts of the Endoscope}

Figure 4 shows the electrical schematic inside the balloon-type vaginal endoscope. All of the electronic components are connected to the control box, which is connected to the handlebar of the endoscope. When the red button is pushed, the light-emitting diode (LED) for system monitoring is on and power is supplied to the system. Data measurements through each sensor are initiated when the power source is supplied and transferred to the Arduino controller. Three depth sensors with infrared (IR) transmitters and receivers are placed at $120^{\circ}$ intervals at the tip of the endoscope. The absolute encoder uses the rotating angle of $\theta$ of the endoscope in the range of $0-360^{\circ}$ and transforms to a value in the range of 0-1024. Additionally, the distance sensor (PSD sensor) used to obtain the distance of $d$ represents the distance from the original coordinate to the manual moving part (including the absolute encoder). The sampling time for each sensor is set to $100 \mathrm{~ms}$.

Figure 5 shows the nozzle design of the endoscope. The detailed description of the displacement of all sensors is also shown. The red rubber ring at the encoder wheel is contacted with the nozzle, and the rotated angle is measured. The mirror at the tip of the nozzle enables us to observe the top view of the nozzle. This means that one camera can observe two-directional image data for the front and top views.

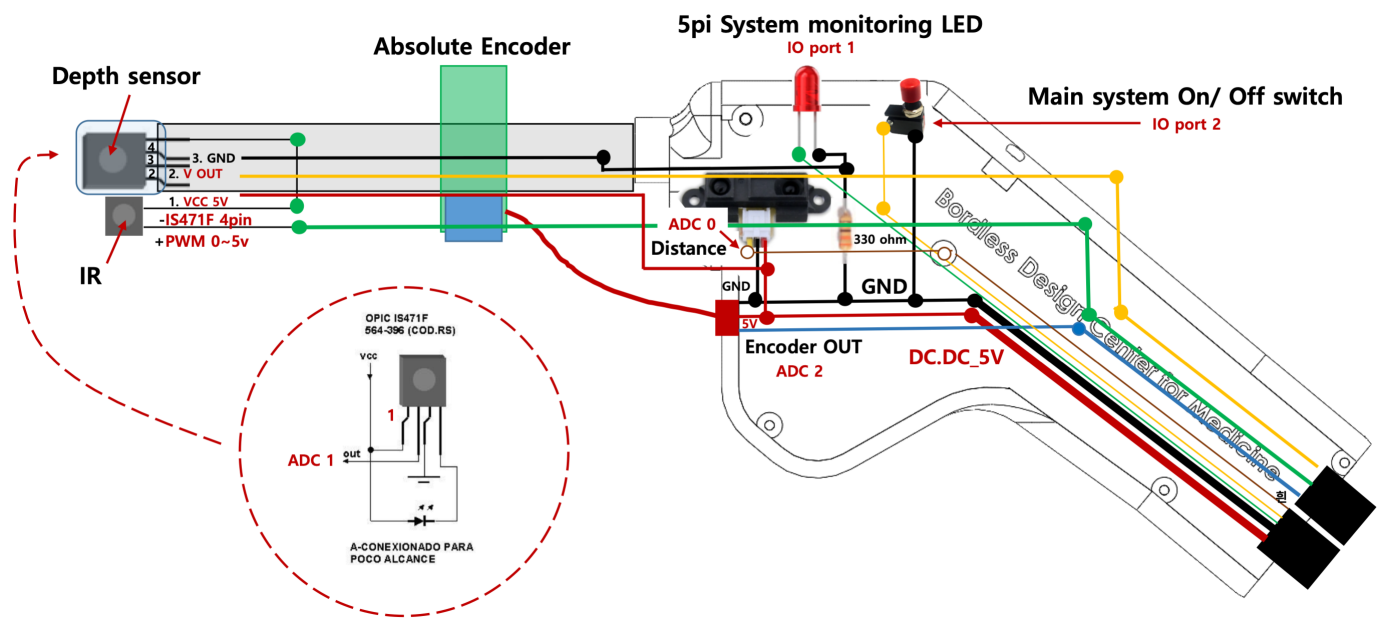

Figure 4. Schematic of the electrical diagram of the balloon-type vaginal endoscope. 


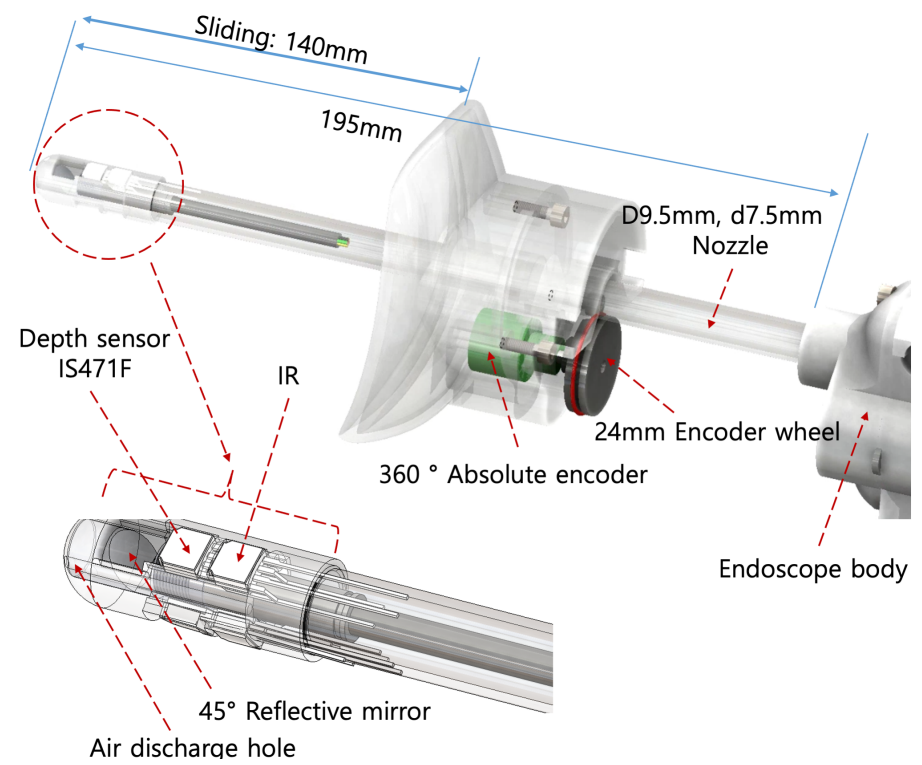

Figure 5. Nozzle design of endoscope.

\subsection{Regulation of the Airflow}

Figure 6 shows the mechanical design of the endoscope based on 3D printing technology by using the 3D computer aided design (CAD) tool of SolidWorks (Dassault Systèmes SolidWorks Corporation, United States of America). The diameter of the nozzle is $9.5 \mathrm{~mm}$, and the distance of aluminum is $210 \mathrm{~mm}$. The 3D printer (3DWOX1 model, Sindoh Co. Ltd., United States of America) makes the total body of fused deposition modeling (FDM) based on the use of polylactic acid (PLA), which is harmless to humans. The thickness of the layer is set to $0.15 \mathrm{~mm}$.

The designed endoscope has the dimensions of distance $\times$ height $\times$ width $=420 \times 175 \times 40 \mathrm{~mm}$, and has a total weight of $400 \mathrm{~g}$. The shape is similar to that of the gun intended for sole use. There is an air cartridge with a $16 \mathrm{~g}$ size and an air on/off valve inside the handlebar. When the valve is working, the injected air is moved to a nozzle through the connected tube and airflow hole at the tip.

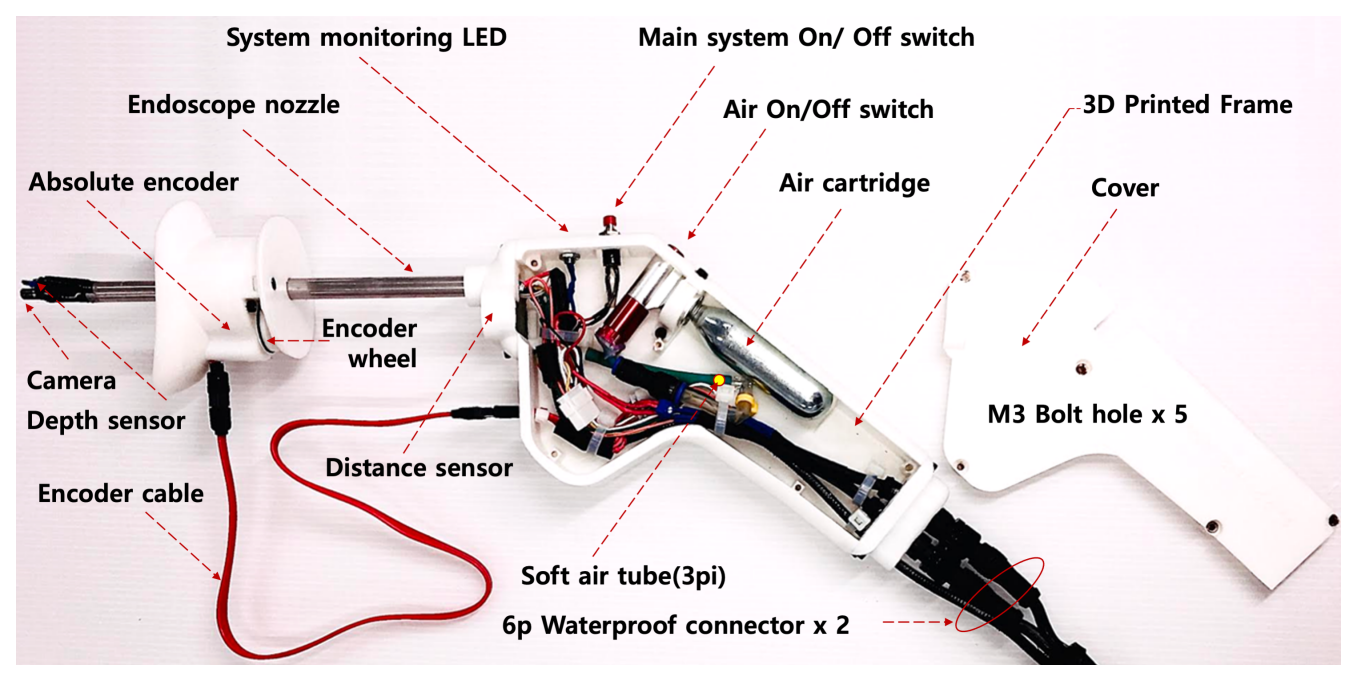

Figure 6. Mechanical design of endoscope based on three-dimensional printing technology.

\section{Results}

\subsection{Results of Design for Testbed}

It is necessary to design the testbed to prove the feasibility of the designed balloon-type vaginal endoscope based on the 3D printing technology. Figure 7 shows the results of the design for the testbed 
based on 3D printing technology to confirm two main points, namely, safe insertion and the ability to acquire exact measurements. Figure $7 \mathrm{a}, \mathrm{b}$ represents the total experimental environment and the results of the actual 3D-printing-based endoscope and testbed, respectively. At first, the hole diameter was set to $40 \mathrm{~mm}$ and the depth length to $140 \mathrm{~mm}$, based on a urologist's advice, to test the safe insertion. After the rubber material is attached to the manual moving part of the endoscope, the rubber's air pressure is improved by the red button at the handlebar. Although it is impossible to decide the appropriate internal air pressure for easy insertion in this experiment, this testbed enables us to test whether it is possible to insert the medical-condom-covered endoscope to the depth of $140 \mathrm{~mm}$ or not. The problem for the proper internal air pressure should be solved in the future with clinical trials.

After the insertion is completed, the performance of the camera and depth sensor is tested. The generator for temporary stimulation is placed on the testbed. The angle of the stimulator and the position of $z_{d}$ are set to $40^{\circ}$ and changed from 20 to $120 \mathrm{~mm}$, respectively. When the stimulator generates an arbitrary prolapse, the feasibility of the exact measurement can be tested to assess whether the camera and depth sensor can measure the arbitrary prolapse. The camera monitor in Figure 7a enables us to observe the internal condition through the camera, and the depth sensor monitor shows the measured data with three depth sensors. It is thought that the testbed is not lacking in the evaluation of the performance and safety of the designed endoscope before the clinical trial.

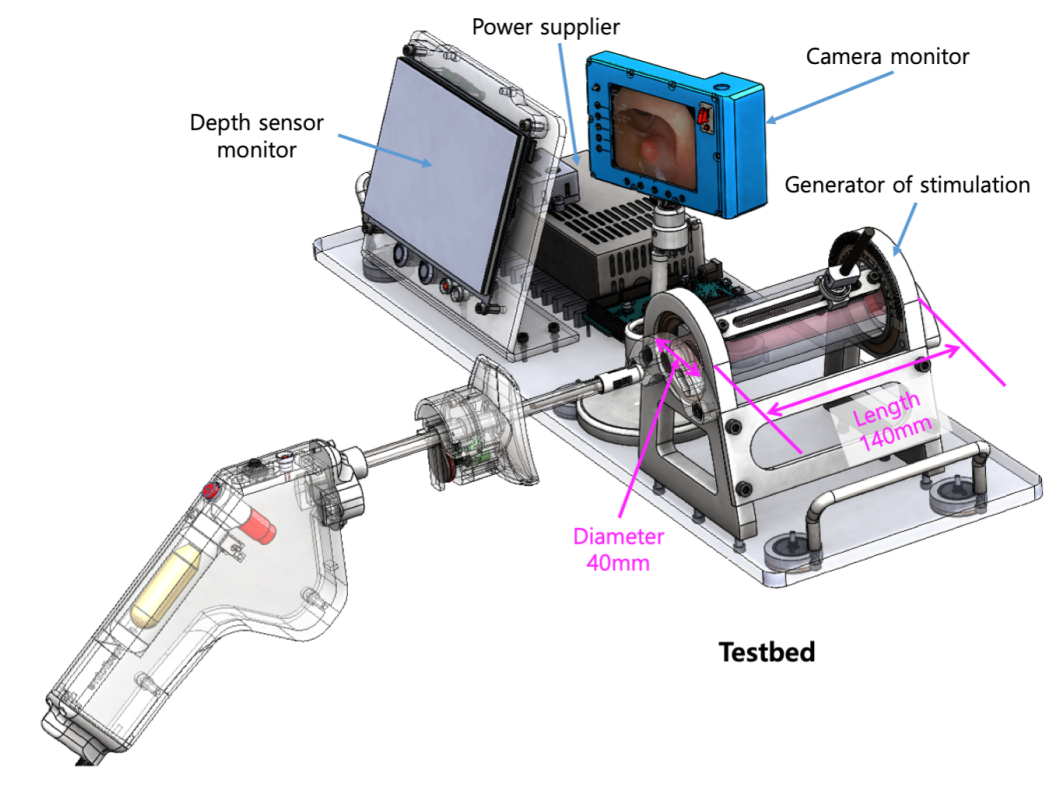

Balloon-type vaginal endoscope

(a) Results of the design for the testbed and the experimental environment.
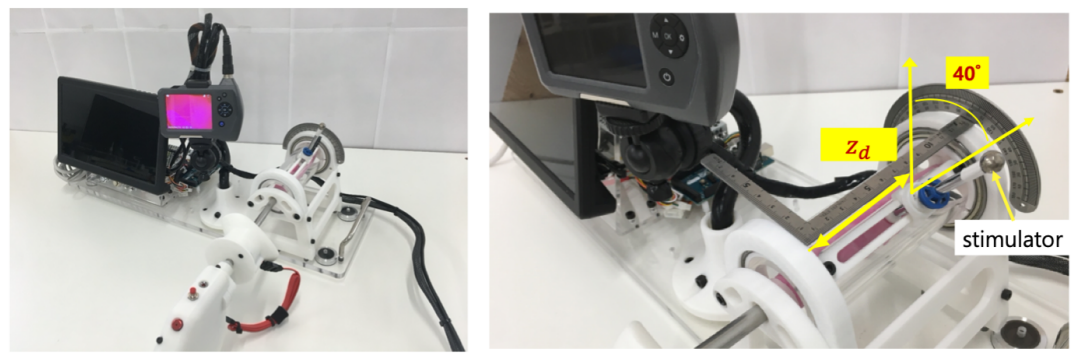

(b) Results of the 3D-printing-based testbed and the location of stimulator.

Figure 7. Design for the testbed based on 3D printing technology to confirm two main points: (a) safe insertion and (b) exact measurement. 


\subsection{Measurement Outcomes for Arbitrary Prolapse through the Testbed}

Figure 8 shows the results of the observed arbitrary prolapse through the camera, subject to the condition wherein the stimulator is forced from the outside of the endoscope: (a) shows the results of the stimulator outside the endoscope, and (b) and (c) show the results of the arbitrary prolapse through the camera (front and top views, respectively). It was found that the small arbitrary prolapse was observed by the camera even if there was a $2 \mathrm{~mm}$ prolapse.

Figure 9 shows the results of the measurements for arbitrary prolapse through the depth sensor, subject to the condition of the stimulator being forced from the outer parts of the endoscope. The horizontal axis indicates the direction of the $z$-axis, and the vertical axis indicates the measured value of $y^{\prime}$ obtained by the depth sensor in Equation (2). This experiment was performed by the $z$-axis directional insertion of the endoscope, subject to the condition of the $110 \mathrm{~mm}$ set of stimulators. It was confirmed that the depth sensor measured the arbitrary prolapse.

As a result, it was proved that it was feasible to identify the prolapse at the early stage with the proposed device, based on the experimental results.

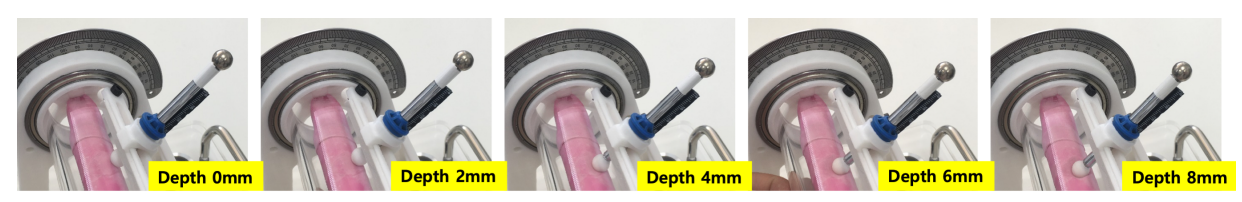

(a) Results of the stimulator outside of endoscope
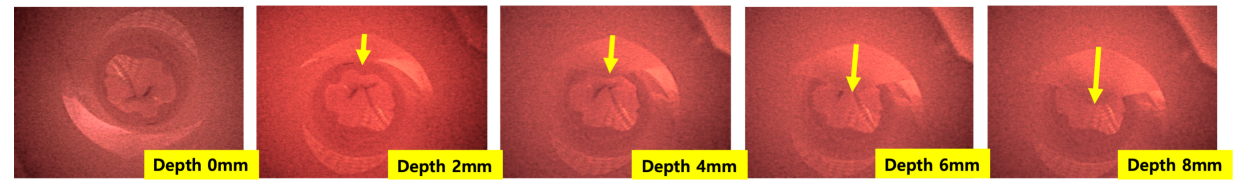

(b) Results of the arbitrary prolapse through the camera (Front view)
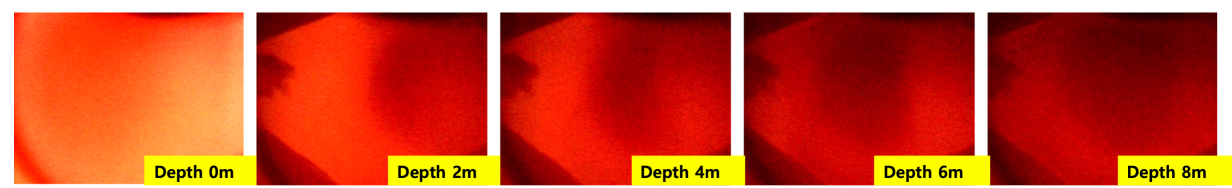

(c) Results of the arbitrary prolapse through the camera (Top view)

Figure 8. Results of observed arbitrary prolapse through the camera, subject to the condition of the stimulator being forced outside the endoscope.

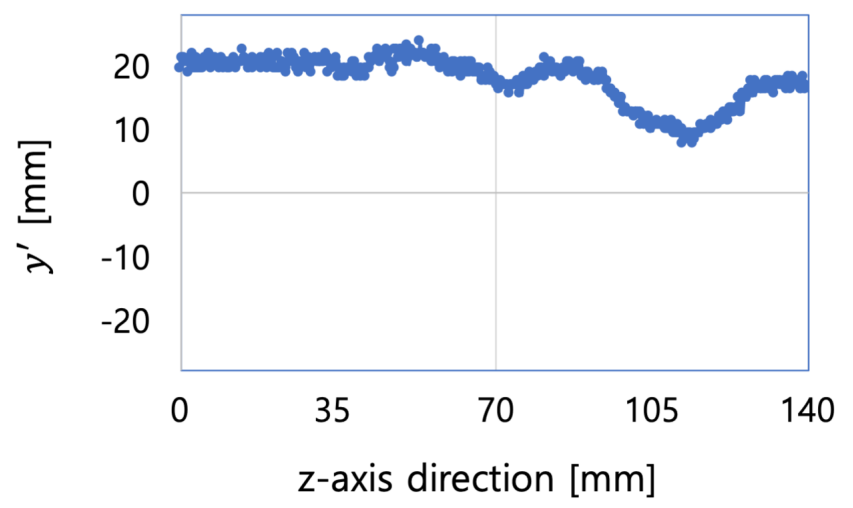

Figure 9. Measurements for arbitrary prolapse through the depth sensor, subject to the condition of the stimulator being forced from the outer parts of the endoscope. 


\section{Discussion}

\subsection{Standardization of Description of Pelvic Organ Prolapse and Specification of System}

It is necessary to describe the present level to perform physical examination findings for system engineers to understand. Although a lot of systems for staging prolapse have been described, the standard is the system approved by the International Continence Society (ICS), the Pelvic Organ Prolapse Quantification (POP-Q) system [17]. The essence of this classification method is that the prescribed anatomical index is used as a fixed reference point. Then, the hymen is a fixed point used as a reference point when measuring six parts. Namely, this system measures nine locations on the vagina and vulva, in centimeters relative to the hymen. These nine locations are used to assign a stage (from 0 to 4 ) of prolapse at its most advanced site. Figure 10a shows the diagrammatic representation of the POP-Q system for staging prolapse by physical examination findings, showing the six sites (points $\mathrm{Aa}$ and $\mathrm{Ba}$ anteriorly, points $\mathrm{Ap}$ and Bp posteriorly, point $\mathrm{C}$ for the cervix or apex, and point $\mathrm{D}$ for the cul-de-sac), genital hiatus (gh), perineal body (pb), and total vaginal length (tvl) used for POP-Q. The prolapse quantification system is probably more detailed than necessary for clinical care, but clinicians should be familiar with it because most studies use the POP-Q system to report research results. A three-by-three grid can be used to concisely organize the measurements as noted in Figure 10b. The stage of the disease, from 0 to 4 , is determined according to the positional relationship of each parameter. Figure 10c is a grid and line diagram contrasting measurements indicating normal support to those of post-hysterectomy vaginal eversion, and Figure 10d is a grid and line diagram representing predominant anterior and posterior vaginal wall prolapse with partial vault descent. This standardization of terminology is useful for system engineers to understand the present level.

Because stage 1 is almost that the edge of prolapse is less than $1 \mathrm{~cm}$, it is possible to find the escaped prolapse through human visual observation. Thus, the main targets for the proposed system are human subjects with stage 0 , to prevent the POP from getting worse. The current specification of the system with a depth length of $14 \mathrm{~cm}$ and a resolution of $1 \mathrm{~mm}$ is enough to measure the POP at the early stage. If not, there is no problem to upgrade the depth length and resolution while replacing the old one with the long-hollow nozzle and high-leveled sensor.

\subsection{Ultrasound Assessment of Pelvic Organ Prolapse}

A pelvic ultrasound is a noninvasive diagnostic exam that produces images that are used to assess organs and structures within the female pelvis. A pelvic ultrasound allows quick visualization of the female pelvic organs and structures including the uterus, cervix, vagina, fallopian tubes, and ovaries. A pelvic ultrasound may be performed in your doctor's office, on an outpatient basis, or as part of your stay in a hospital. Procedures may vary depending on your condition and your hospital's practices. Although there have been a lot of studies performed, little information is available to help define whether a certain degree of pelvic organ prolapse is clinically relevant. Dietz et al. [31] reported that descent of the bladder to $\geq 10 \mathrm{~mm}$ and of the rectum to $\geq 15 \mathrm{~mm}$ below the symphysis pubis are strongly associated with symptoms, and these values are proposed as cut-offs for the diagnosis of significant prolapse based on receiver operating characteristics statistics.

Compared with this, the proposed camera-and-depth-sensors-based system is a point-of-care device for healthcare. Although the ultrasound device is for diagnosis in the hospital, the user can observe the location at any time by herself when feeling something strange. Furthermore, it is necessary to study particular human anatomy to understand imaging data from the ultrasound device; however, there is no need to have the knowledge and skills in a field related to the medical major when the proposed system is used. 


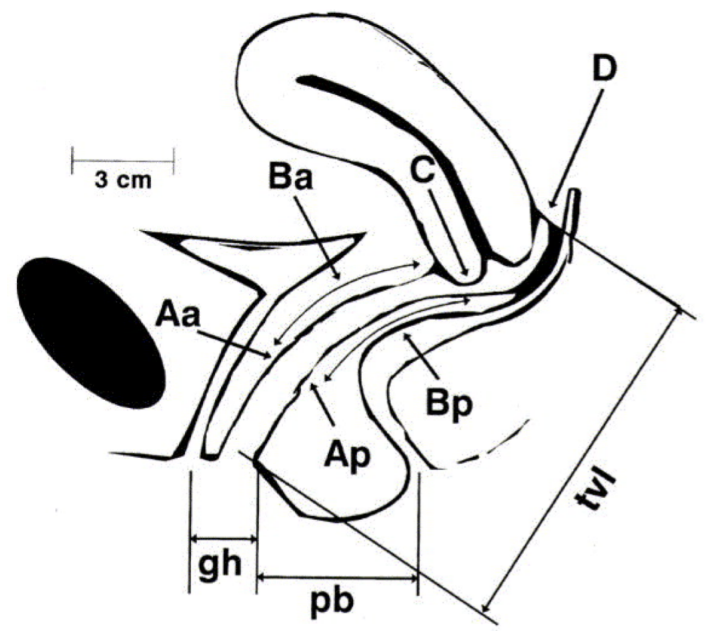

(a) Six sites (points $A a, B a, C, D, B p$, and Ap), genital hiatus (gh), perineal body (pb), and total vaginal length (tvl) used for pelvic organ support quantitation.

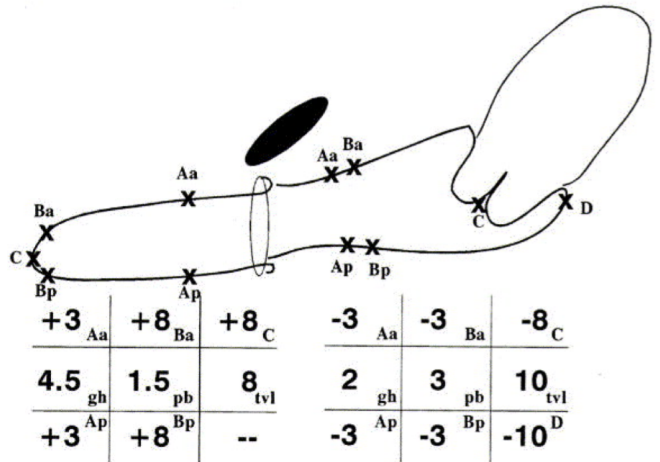

A

B

(C) A, Complete eversion of vagina - stage 4 prolapse. B, Normal support that represents stage 0 support.

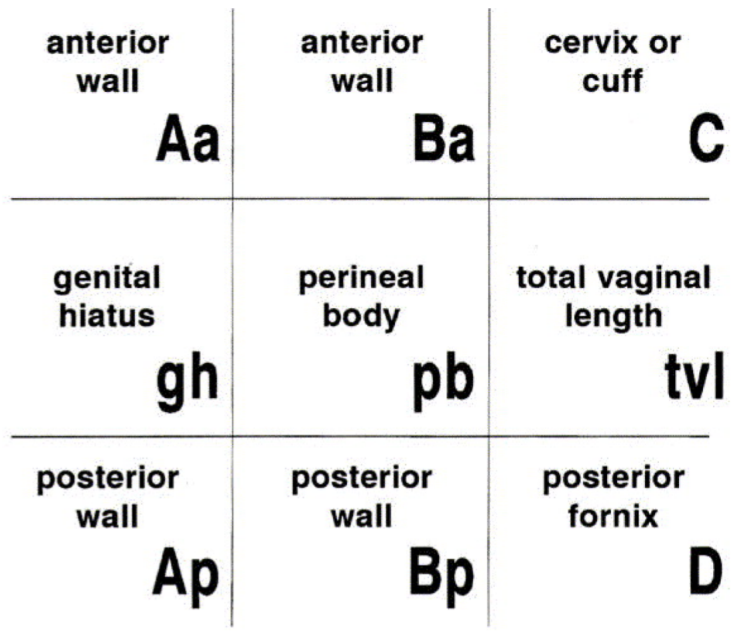

(b) Three-by-three grid for recording quantitative description of pelvic organ support.

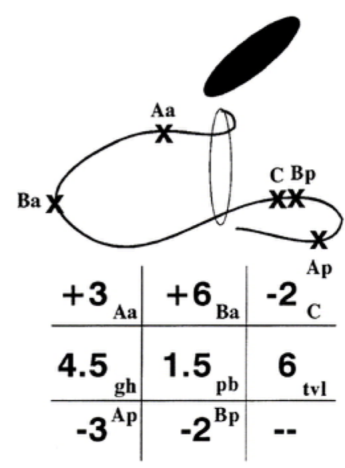

A

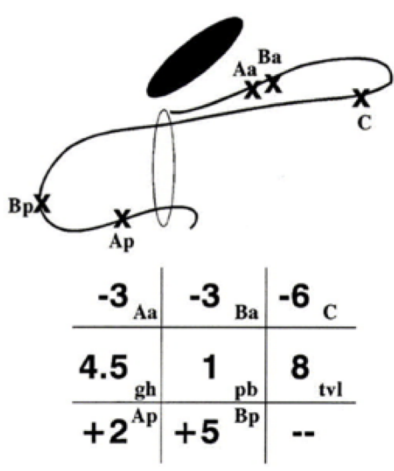

B

(d) A, Predominant anterior support defect that represents stage 3 Ba prolapse. B, Predominant posterior support defect that represents stage $3 \mathrm{Bp}$ prolapse.

Figure 10. Standardization of terminology and four diagnostic examples. Reproduced from Bump RC, Mattiasson A, Bo K, Brubaker LP, DeLancey JO, Klarskov P, Shull B, Smith A. The standardization of terminology of female pelvic organ prolapse and pelvic floor dysfunction. Am J Obstet Gynecol 1996;175:10-17. Copyright 1996, with permission form Elsevier [17].

\section{Conclusions}

Over the past few years, the adopted medical approach for the diagnosis of female vaginal- and pelvic-related diseases has changed [32]. Many urologists treat more patients with POP than with urinary incontinence. Some patients have only urinary incontinence, but there are even more patients with multiorgan problems, including urinary and fecal incontinence, and POP. According to this change, it is the improved point of this study that continuous observation through a 3D-printing-based vaginal endoscope helps prevent POP. Furthermore, it is an excellent strategy to allow female patients to check themselves and improve the inconveniences they feel with pelvic exercises and pessary insertion when they feel something wrong. Finally, it seems that the active response based on direct observations through the camera enables us to decide the types and the position/orientation of the pessaries. 
Author Contributions: Conceptualization, Formal Analysis, Investigation, and Writing-Original Draft Preparation: H.J. and M.J.; Writing-Review and Editing, M.E. and M.K.; Project Administration, M.E. and Y.O. All authors have read and agreed to the published version of the manuscript.

Funding: This study was financially supported by Chonnam National University (Grant number: 2020-2020) and partially supported by the fund supporting International Joint Lab-Type A in Osaka University.

Acknowledgments: We gratefully acknowledge the work of past and present members of our laboratory at Osaka University. Special thanks go to Takafumi Ohno for his administrative support.

Conflicts of Interest: The authors declare no conflict of interest.

\section{References}

1. Dietz, H.P. Pelvic organ prolapse-A review. Aust. Fam. Physician 2015, 44, 446. Title Cited Article: J. Abbr. 2008, 10, 142-149.

2. U.S. Census Bureau. U.S. Interim Projections by Age, Sex, Race, and Hispanic Origin. Available online: https://www2.census.gov/programs-surveys/popproj/technical-documentation/methodology/ idbsummeth.pdf (accessed on 5 June 2020).

3. Luber, K.M.; Boero S.; Choe J.Y. The demographics of pelvic floor disorders: Current observations and future projections. Am. J. Obstet. Gynecol. 2001, 184, 1496-1503. [CrossRef] [PubMed]

4. Subak, L.L.; Waetjen, L.E.; van den Eeden, S.; Thom, D.H.; Vittinghoff, E.; Brown, J.S. Cost of pelvic organ prolapse surgery in the United States. Obstet. Gynecol. 2001, 98, 646-651. [PubMed]

5. Glazener, C.; MacArthur, C.; Bain, C.; Dean, N.; Toozs-Hobson, P.; Richardson, K.; Lancashire, R.; Herbison, P.; Hagen, S.; Grant, A.; et al. Epidemiology of pelvic organ prolapse in relation to delivery mode history at 12 years after childbirth: A longitudinal cohort study. Neurourol. Urodyn. 2010, 29, 819-820.

6. Patel, D.; Xu, X.; Thomason, A.D.; Ransom, S.B.; Ivy, J.S.; DeLancey, J.O. Childbirth and pelvic floor dysfunction: An epidemiologic approach to the assessment of prevention opportunities at delivery. Am. J. Obstet. Gynecol. 2006, 195, 23-28. [CrossRef] [PubMed]

7. Young, N.; Kamisan Atan, I.; Dietz, H. Obesity: How much does it matter for female pelvic organ prolapse? In RCOG World Congress; RCOG: Brisbane, Australia, 2015.

8. Abdool, Z.; Dietz, H. A comparison of functional pelvic floor anatomy in white and black South African women with symptomatic pelvic organ prolapse. Neurourol. Urodyn. 2014, 33, 910-911.

9. Laborda, E.; Gelman, W.; Anthony, F.; Monga, A. Is increased collagen metabolism the cause or effect of prolapse: A controlled study. Neurourol. Urodynam. 2003, 22, 505-506.

10. Phillips, C.H.; Anthony, F.; Benyon, C.; Monga, A.K. Collagen metabolism in the uterosacral ligaments and vainal skin of women with uterine prolapse. BJOG 2006, 113, 39-46. [CrossRef]

11. Ellerkmann, R.M.; Cundiff, G.W.; Melick, C.F.; Nihira, M.A.; Leffler, K.; Bent, A.E. Correlation of symptoms with location and severity of pelvic organ prolapse. Am. J. Obstet. Gynecol. 2001, 185, 1332-1338. [CrossRef]

12. Heit, M.; Culligan, P.; Rosenquist, C.; Shott, S. Is pelvic organ prolapse a cause of pelvic or low back pain? Obstet. Gynecol. 2002, 99, 23-28.

13. Handa, V.L.; Harvey, L.; Cundiff, G.W.; Siddique, S.A.; Kjerulff, K.H. Sexual function among women with urinary incontinence and pelvic organ prolapse. Am. J. Obstet. Gynecol. 2004, 191, 751-756. [CrossRef]

14. Ulrich, D.; Guzman Rojas, R.; Dietz, H.P.; Mann, K.; Trutnovsky, G. Use of a visual analog scale for evaluation of bother from pelvic organ prolapse. Ultrasound Obstet. Gynecol. 2014, 43, 693-697. [CrossRef] [PubMed]

15. Barber, M.D. Symptoms and outcome measures of pelvic organ prolapse. Clin. Obstet. Gynecol. 2005, 48, 648-661. [CrossRef] [PubMed]

16. Dietz, H.P.; Haylen, B.T.; Vancaillie T.G. Female pelvic organ prolapse and voiding function. Int. Urogynecol. J. 2002, 13, 473-474. [CrossRef] [PubMed]

17. Bump, R.C.; Mattiasson, A.; Bo, K.; Brubaker, L.P.; DeLancey, J.O.; Klarskov, P.; Shull, B.L.; Smith, A.R. The standardization of terminology of female pelvic organ prolapse and pelvic floor dysfunction. Am. J. Obstet. Gynecol. 1996, 175, 10-17. [CrossRef]

18. Weber, A.M.; Richter, H.E. Pelvic Organ Prolapse. Obstet. Gynecol. 2005, 106, 615-634. [CrossRef]

19. Persu, C.; Chapple, C.R.; Cauni, V.; Gutue, S.; Geavlete, P. Pelvic Organ Prolapse Quantification System (POP-Q)-A new era in pelvic prolapse staging. J. Med. Life 2011, 4, 75-81. 
20. Diez, H.P.; Shek, K.L. Levator defects can be diagnosed by 2D translabial ultrasound. Int. Urogynecol. J. 2009, 20, 807-811.

21. Paraiso, M.F.; Walters, M.D.; Rackley, R.R.; Melek, S.; Hugney, C. Laparoscopic and abdominal sacral colpopexies: A comparative cohort study. Am. J. Obstet. Gynecol. 2005, 192, 1752-1758. [CrossRef]

22. Visco, A.G.; Weidner, A.C.; Barber, M.D.; Myers, E.R.; Cundiff, G.W.; Bump, R.C.; Addison, W.A. Vaginal mesh erosion after abdominal sacral colpopexy. Am. J. Obstet. Gynecol. 2001, 184, 297-302. [CrossRef]

23. Olsen, A.L.; Smith, V.J.; Bergestrom, J.O.; Colling, J.C.; Clark, A.L. Epidemiology of surgically managed pelvic organ prolapse and urinary incontinence. Obstet. Gynecol. 1997, 89, 501-506. [CrossRef]

24. Bent, A.E.; Cundiff, G.W.; Swift, S.E. Ostergard's urogynecology and pelvic floor dysfunction. In Chapter 26 Surgical Management of Pelvic Organ Prolapse; Lippincott Williams \& Wilkins: Philadelphia, PA, USA, 2008.

25. Thakar, R.; Stanton S. Management of genital prolapse. BMJ 2004, 324, 1258-1262. [CrossRef] [PubMed]

26. Harvey, M.A. Pelvic floor exercises during and after pregnancy: A systematic review of their role in preventing pelvic floor dysfunction. J. Obstet. Gynaecol. Can. 2003, 25, 487-498. [CrossRef]

27. Cundiff, G.W.; Weidner, A.; Visco, A.G.; Bump, R.C.; Addison, W.A. A survey of pessary use by the membership of the American Urogynecology Society. Obstet. Gynecol. 2000, 95, 931-935. [PubMed]

28. Pott-Grinstein, E.; Newcomer, J.R. Gynecologists' patterns of prescribing pessaries. J. Reprod. Med. 2001, 46, 205-208. [PubMed]

29. Clemons, J.L.; Aguilar, V.C.; Tillinghast, T.A.; Jackson, N.D.; Myers, D.L. Risk factors associated with an unsuccessful pessary fitting trial in women with pelvic organ prolapse. Am. J. Obstet. Gynecol. 2004, 190, 345-350. [CrossRef]

30. Clemons, J.L.; Aguilar, V.C.; Tillinghast, T.A.; Jackson, N.D.; Myers, D.L. Patient satisfaction and changes in prolapse and urinary symptoms in women who were fitted successfully with a pessary for pelvic organ prolapse. Am. J. Obstet. Gynecol. 2004, 190, 1025-1029. [CrossRef]

31. Dietz, H.P.; Lekskulchai, O. Ultrasound assessment of pelvic organ prolapse: The relationship between prolapse severity and symptoms. Ultrasound Obstet. Gynecol. 2007, 29, 688-691. [CrossRef]

32. Kobashi, K.C.; Leach, G.E. Pelvic prolapse. J. Urol. 2000, 164, 1879-1890. [CrossRef]

(C) 2020 by the authors. Licensee MDPI, Basel, Switzerland. This article is an open access article distributed under the terms and conditions of the Creative Commons Attribution (CC BY) license (http:/ / creativecommons.org/licenses/by/4.0/). 